\author{
B. $\mathrm{HOU}^{1, \varpi}$ \\ J. NEES ${ }^{1}$ \\ A. MORDOVANAKIS ${ }^{1}$ \\ M. WILCOX ${ }^{1}$ \\ G. MOUROU ${ }^{1}$ \\ L.M. CHEN $^{2}$ \\ J.-C. KIEFFER ${ }^{2}$ \\ C.C. CHAMBERLAIN ${ }^{3}$ \\ A. $\mathrm{KROL}^{3}$
}

\title{
Hard X-ray generation from solids driven by relativistic intensity in the lambda-cubed regime
}

\author{
${ }^{1}$ Focus Center and Center for Ultrafast Optical Science, University of Michigan, 2200 Bonisteel Boulevard, \\ Ann Arbor, MI 48109-2099, USA \\ 2 INRS-énergie et Matériaux, Université du Québec, Varennes, Québec, 1650 Montée Sainte-Julie, \\ J3X 1S2, Canada \\ ${ }^{3}$ SUNY Upstate Medical University, Department of Radiology, 750 E. Adams. St., \\ Syracuse NY 13210, USA
}

\section{Received: 26 Ocotober 2005}

Published online: 10 January 2006 • C Springer-Verlag 2005

ABSTRACT Interaction of relativistic intensity laser pulses encompassed at focus by a volume of a few wavelengths cubed with solids is examined. Spectroscopy of hard X-rays of several metallic targets, including $\mathrm{Cu}, \mathrm{Ge}, \mathrm{Mo}, \mathrm{Ag}$, and $\mathrm{Sn}$, irradiated in this regime at a high repetition rate $(0.4 \mathrm{kHz})$, has been experimentally studied. The $K_{\alpha}$ and $K_{\beta}$ peaks of all targets were obtained. Averaged electron temperatures of several tens of $\mathrm{keV}$ and total X-ray conversion efficiencies up to $0.02 \%$ are calculated. The X-ray source size is measured to be $\sim 10$ micron with varying elliptical shape.

PACS 52.38.Ph; 52.59.Px; 52.70.La

\section{1}

\section{Introduction}

Over the past 15 years, the laser intensity available to experimentalists has increased many orders of magnitude. Aided chiefly by chirped pulse amplification [1,2] (CPA), a large number of labs have been able to reach intensities well in the relativistic range $\left(2 \times 10^{18} \mathrm{~W} / \mathrm{cm}^{2}\right.$ at $800 \mathrm{~nm}$ wavelength). At such intensities, the electron quiver energy due to the laser field is comparable with its rest mass energy. This capability has opened a floodgate of experimental results, demonstrating the acceleration of electrons [3-5] and ions [6-8] to high energies and the production of X-rays [9$11], \gamma$-rays [12,13], and positrons [14, 15]. These high intensities have also made it possible to observe relativistic nonlinear effects like relativistic self-focusing $[16,17]$ and harmonic generation [18] in solids.

$\mathrm{X}$-ray generation from the interaction of ultraintense laser pulses with solids is a widely studied subject in the area of high field physics. When intense laser fields are incident on a solid surface, an inhomogeneous, and dense plasma is formed. During the interaction of the laser with the solid or preformed plasma, a part of the laser energy can be coupled into suprathermal (hot) electrons through resonance absorption, vacuum heating, and $\boldsymbol{J} \times \boldsymbol{B}$ heating [19]. Many of the hot electrons leave the dense-plasma region penetrating forward into the bulk material or are emitted backward into the vacuum. Consequently a strong space-charge field is set up within

Fax: 1-734-763-4876, E-mail: houbx@eecs.umich.edu the plasma. This electric field draws back a significant part of the backward-emitted hot electrons, many of which penetrate into the "cold" target. This produces X-ray pulses which spectrally consist of continuous bremsstrahlung and characteristic line emission. The former ranges from several tens of $\mathrm{eV}$ to a few $\mathrm{MeV}$ depending on the parameters of the laser and the plasma, whereas the latter can be $1 \mathrm{keV}-100 \mathrm{keV}$ depending on the target material. For ultrashort laser pulses, the $\mathrm{X}$-ray pulse durations generally range from a few hundreds of femtoseconds to several picoseconds [20,21], and X-ray source size can be a few times larger than the laser spot size. Such ultrafast X-ray sources have been used in many experiments, such as, time resolved diffraction, medical imaging, spectroscopy and microscopy of transient physical, chemical, or biological phenomena [22-25].

For this kind of X-ray sources to be more widely used, the laser system must be more compact, while keeping a high focal intensity with less pulse energy. Low pulse energy also allows high repetition rate operation. Two parameters of the pulse can be manipulated to reach this purpose, shortening the pulse durations to few cycles and reducing the focal spot size to the near-single wavelength scale. At these limits, all the pulse energy is assembled in a "football" with dimensions on the scale of $\lambda$. We call the laser operating in this regime $a \lambda^{3}$ laser. The potential for enhancing the utility of laser based sources and for investigating laser-matter interaction in this small volume $[26,27]$ is the basis of our recent work. This $\lambda^{3}$ concept also applies to Joule-range laser systems and light with shorter wavelengths.

Numerous experiments on hard X-ray generation from the interaction of intense light with solids have been conducted [9-11,28-31] with relativistic intensities. The laser systems used in these experiments typically operate at low repetition rates (single shot to $10 \mathrm{~Hz}$ ) in the Joule range. High repetition rate lasers $(\mathrm{kHz})$ in the low milli-Joule range were also used by a few groups [32-34] with focal intensities ranging from $10^{16}-10^{17} \mathrm{~W} / \mathrm{cm}^{2}$. On the other hand, milliJoule pulse can be concentrated in the $\lambda^{3}$ regime to obtain relativistic intensities. This can be accomplished by using few- to several-cycle pulses focused to single-wavelength transverse dimensions. The small focal spot makes it possible to produce small X-ray sources, while the short laser pulse duration gives rise to short X-ray pulses. We have shown that the relativistic intensity regime can be reached with a laser in the milli-Joule 
range at $\mathrm{kHz}$ repetition rates [35]. The pulse was less than 8 periods in duration and had a 1.6- $\mu \mathrm{m}$ spot size. Alternately, these pulses have also been directed through a hollow-core fiber and chirped mirror compressor to produce $<10$-fs $(\sim 3$ cycles) pulses with $0.2 \mathrm{~mJ}$ energy that have a $1.2 \times 1.4-\mu \mathrm{m}^{2}$ focus and relativistic intensity [36].

In this paper, we present the results of laser-matter interaction in the relativistic $\lambda^{3}$ regime. We have studied the spectroscopy of hard X-rays from several metallic targets, including $\mathrm{Cu}, \mathrm{Ge}, \mathrm{Mo}, \mathrm{Ag}$, and $\mathrm{Sn}$, using a single-stage multipass amplifier at $0.4 \mathrm{kHz} . K_{\alpha}$ and $K_{\beta}$ peaks of all targets were obtained. Averaged electron temperatures of several tens $\mathrm{keV}$ and total X-ray conversion efficiencies up to $0.02 \%$ are measured. The X-ray source size is measured to be $\sim 10$ micron with varying elliptical shape. These smaller sources potentially have better spatial coherence.

\section{2}

\section{Experiment setup}

The experiments were performed with a compact table-top Ti:sapphire laser system, consisting of an oscillator, an all-reflective grating stretcher, a single-stage multi-pass amplifier and a grating-pair compressor. The system similar to that described in [35], but without the second stage of amplification, produces a train of pulses with $2.3-\mathrm{mJ}$ pulse energy, giving $22 \mathrm{fs}$ (8-cycles) and $1.3 \mathrm{~mJ}$ after compression. A fast Pockels cell (Medox Electro-Optics/Thales) was installed between the amplifier and the compressor, to increase nanosecond prepulse-contrast to $10^{6}$. This also protects the amplifier from damage due to retro-reflected light from the plasma.

A very important element in producing ultrahigh intensity from this compact laser system is to correct the laser's wave-front distortion and to pre-compensate for the aberrations caused by the focusing optic, an $f / 1$ off-axis $\left(60^{\circ}\right)$ paraboliodal mirror. This is accomplished in our setup by a 47-mm-diameter, silver-coated deformable mirror (DM) (Xinetics Inc.) with 37 computer-controlled (OKO Technologies) electrostrictive actuators. This arrangement allows us to produce a $\lambda^{2}$ focal area with minimal background. Measurement of the focal spot with a $60 \times$ microscope-objective and a 16-bit charge-coupled-device (CCD) camera indicates that the focal spot is $1.2 \times 1.4 \mu \mathrm{m}^{2}$, full width at half maximum. With 0.8 -mJ, 22-fs pulses on target, the intensity is estimated to reach the relativistic intensity, $2.2 \times 10^{18} \mathrm{~W} / \mathrm{cm}^{2}$ in the $\lambda^{3}$ regime with a focal volume of $<(3 \lambda)^{3}$. This intensity was confirmed by ion time-of-flight spectroscopy experiment, in which $\mathrm{Ar}^{11+}$ was observed in low density Ar gas under highest pulse energy, indicating focused intensity $\sim 2 \times 10^{18} \mathrm{~W} / \mathrm{cm}^{2}$. After averaging the focal spot for 5 minutes $\left(10^{5}\right.$ shots $)$, no measurable change of image size was found, indicating good pointing stability.

The experimental set-up is shown in Fig. 1. Laser pulses of 47-mm-diameter aperture from the DM are transported into the vacuum chamber through a 3-mm-thick fused silica window, where they are then focused by the paraboloid onto the various targets. These targets are polished disks with thickness of $10-15 \mathrm{~mm}(\mathrm{Cu}, \mathrm{Mo}, \mathrm{Ag}$, and $\mathrm{Sn})$ or polished wafer $(\mathrm{Ge})$ that are rotated and translated to expose a fresh area to each laser shot in a spiral pattern. $p$-polarized relativistic-

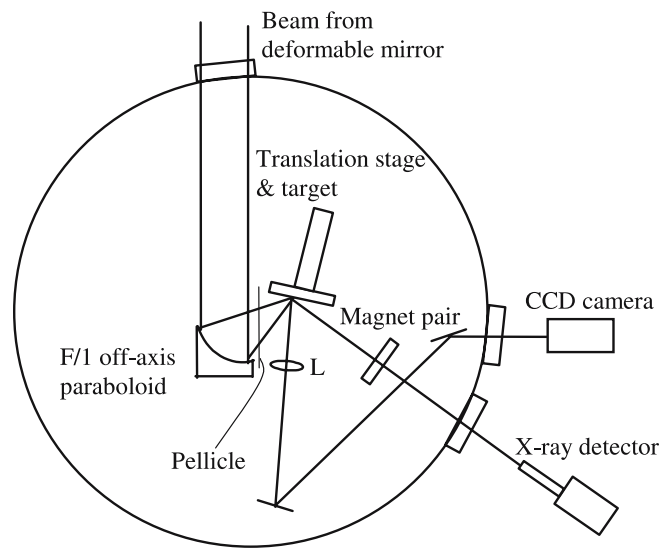

FIGURE 1 Schematic of the experimental setup

intensity pulses are incident on the targets at $45^{\circ}$. The resulting X-ray spectra are measured with an X-ray detector (XR-100CZT/, Amptek, Inc.), $52 \mathrm{~cm}$ from the source, at an angle of $40^{\circ}$ from the target normal (on the side opposite the incident beam). Its output signal is amplified (in a PX2TCZT amplifier, Amptek, Inc.) and recorded by a multi-channel analyzer. The detector system has a spectral cut-off around $4 \mathrm{keV}$ due to the threshold setting on the multi-channel analyzer. The detector response is nearly uniform up to approximately $100 \mathrm{keV}$, and is calibrated using an ${ }^{241} \mathrm{Am}$ source. To avoid the pile-up effect, a $150-\mu \mathrm{m}$ or $90-\mu \mathrm{m}$ lead pinhole is placed $5.5 \mathrm{~cm}$ in front of the detector. A pair of magnets is placed between the plasma source and the beryllium $(\mathrm{Be})$ chamber window to deflect high-energy electrons, thus preventing X-ray fluorescence in the Be window. A lens, $\mathrm{L}$, in the chamber images the plasma plume onto a CCD camera to monitor the target position in the focal region. Care is taken to align the polished target surface accurately due to the short Rayleigh range $\left(2 z_{\mathrm{r}}=13 \mu \mathrm{m}\right)$ of the high-numerical-aperture focusing optic. Because the $f / 1$ paraboloid is very sensitive to the alignment of the beam, we optimize the DM under the vacuum of the experimental condition $\left(5 \times 10^{-4} \mathrm{mbar}\right)$ in order to compensate for potential vacuum-induced mechanical distortions. A 2- $\mu \mathrm{m}$ thick nitrocellulose pellicle (National Photocolor) is used to protect the paraboloid from the debris produced by target ablation. The spectra are accumulated over $\sim 10^{5}$ laser shots.

\section{$3 \quad$ X-ray spectra}

The X-ray spectra generated in our experiments are shown in Fig. 2. It is clear that the characteristic line spectra are superimposed on broadband continua from $4 \mathrm{keV}$ to $65 \mathrm{keV}$. The observed intensity ratio between the $K_{\alpha}$ peak and background decreases with increasing atomic number, $Z$. There are two reasons for this effect. First, bremsstrahlung emission is proportional to $Z^{2}$ [37]. Second, as atomic number increases, $K$-shell ionization energy increases. This leads to a reduction of the $K$-shell ionization cross-section by electron impact [38]. Thus, $K$-shell emission decreases as $Z$ increases for a given electron energy distribution. The $K_{\alpha}$ and $K_{\beta}$ peaks for all targets can be seen in the respective insets. The slight pile-up effect in the spectra of $\mathrm{Cu}$ and $\mathrm{Ge}$ can be seen by 


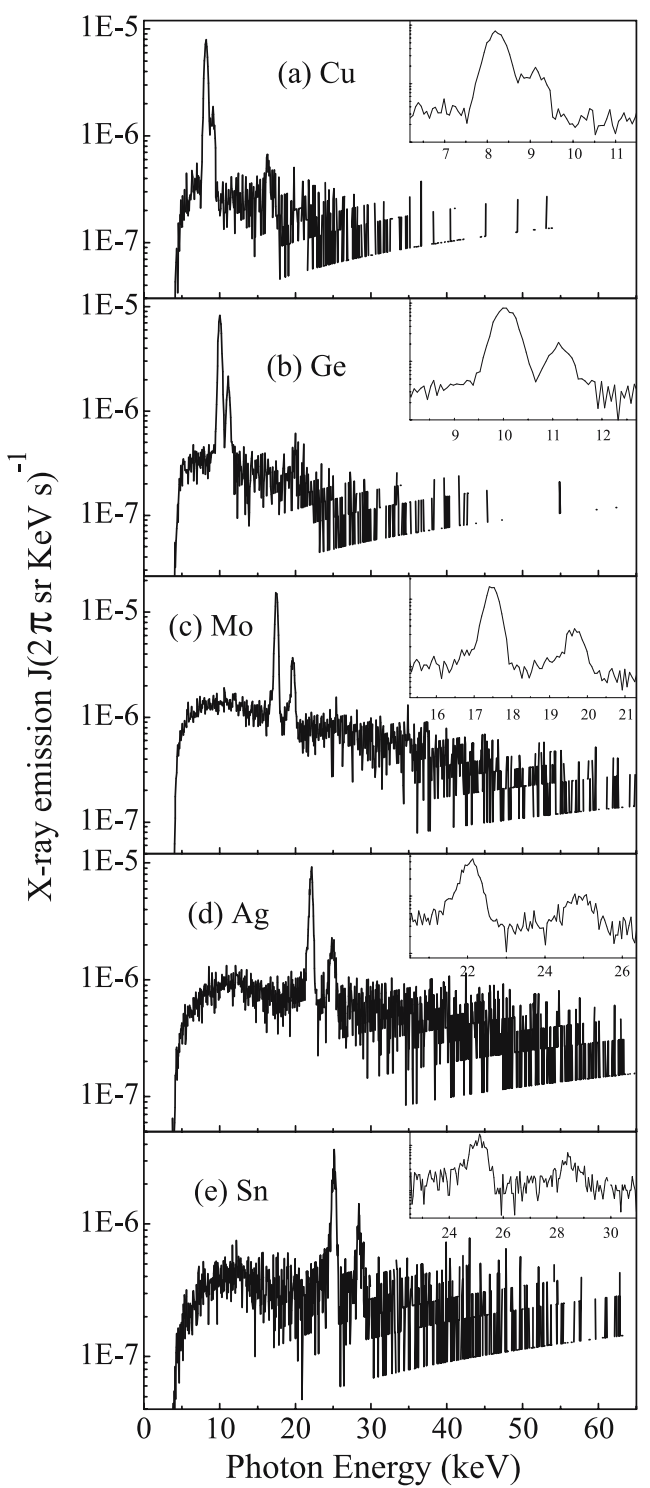

FIGURE $2 X$-ray spectra obtained in the experiments. (a) $\mathrm{Cu}$, (b) $\mathrm{Ge}$, (c) Mo, (d) Ag, and (e) $\mathrm{Sn}$

a small peak at twice the $K_{\alpha}$ line energy. The energies of these observed characteristic lines are in excellent agreement with energy levels of $k$-shell emission from neutral elements found in the literature [38].

From the X-ray spectra measured in our experiments, we can estimate the energy conversion efficiency from laser to total X-ray energy (continuum emission plus line emission), and the energy conversion efficiencies from laser to characteristic line emissions. The conversion efficiencies shown in Table 1 are for the X-rays in the $2 \pi$ sr solid angle in the front of the target, using the nearly isotropic X-ray distribution indicated by angularly resolved measurements using the same detector. The conversion efficiencies for Mo and Ag emission are the highest for the targets tested. Their superiority over the other materials may result from their superior surface flatness and low wobble $( \pm 0.75 \mu \mathrm{m})$. While the $S n$ target has a surface flatness as good as Mo and Ag, its plume deposits more debris, reducing the pellicle transmittance during exposure and lowering the accumulated X-ray signal. The Ge wafer target dis- plays larger surface warp that allows the surface to move out of ideal focus, possibly lowering conversion efficiency. With $0.02 \%$ total conversion efficiency, the Mo and Ag sources are among the most efficient laser-based hard X-ray sources. Mo $K_{\alpha}$ emission is generated with $0.004 \%$ efficiency; nearly as efficient as previous work [30] generating $\mathrm{Cu} K_{\alpha}$, but here we have twice the photon energy and it is emitted into half the solid angle. For the Mo target, the X-ray flux of $K_{\alpha}$ emission is $3.1 \times 10^{9}$ photons $/(2 \pi \mathrm{srs})$, and the total $\mathrm{X}$-ray flux is $1.7 \times$ $10^{10}$ photons $/(2 \pi \mathrm{srs})$, or $7.8 \times 10^{6}$ photons $/(2 \pi \mathrm{srshot})$ and $4.3 \times 10^{7}$ photons $/(2 \pi$ sr shot $)$, respectively.

From the bremsstrahlung background of our time-integrated spectra, it can be seen that there is a single exponential decay of X-ray spectral power with photon energy in our detection window (from 4 to $65 \mathrm{keV}$ ). This implies a MaxwellBoltzmann-like electron distribution [37] in this range. The temperature associated with the electrons in our experiments can then be calculated from the slope of the X-ray spectra. The calculated results are shown in Table 1, which range from 16 to $48 \mathrm{keV}$. Electron temperatures for $\mathrm{Ag}$ and $\mathrm{Sn}$ targets are significantly higher than they are for the other targets. These were prepared with a non-specular surface finish, as opposed to $\mathrm{Cu}, \mathrm{Ge}$, and $\mathrm{Mo}$, which had a mirror-like finish. The target's surface roughness can enhance the laser energy absorption, consequently the electron temperature and conversion efficiency. Our observations may be similar to those described in the literature [39].

\section{$4 \quad$ X-ray source size}

Knife-edge measurements were used to determine $\mathrm{X}$-ray source size. The $\mathrm{X}$-ray source images a knife-edge onto Kodak DEF film with a magnification of $\sim 40$. The DEF film is installed at the position of the X-ray detector in Fig. 1. The edge spread function can be obtained from this image, which is calibrated for linearity with visible light using a standard sensitometer-densitometer calibration procedure. The edge spread function is fitted to a Fermi function $\mathrm{F}(x)=a+$ $b /[\exp (x-c) / d+1]$. The line-spread function is then produced by differentiating the Fermi function. The width of the Gaussian fit of this line spread function indicates the source size, taking into account the geometric magnification. Considering that the knife edge is not perfectly straight, we analyze a single line (perpendicular to the edge) of pixels on the image, and repeat this process for each line. The averaged result is reported. We use horizontal- and vertical-edge projection to measure the vertical and horizontal source sizes, respectively.

For $\mathrm{Cu}, \mathrm{Mo}, \mathrm{Ag}$, and $\mathrm{Sn}$ targets, the measured X-ray source sizes are shown in Table 1. The vertical size ranges from $7.3 \mu \mathrm{m}$ to $10.3 \mu \mathrm{m}$, and the horizontal ranges from $10.4 \mu \mathrm{m}$ to $15.0 \mu \mathrm{m}$. We can see that all the sources are elliptical. The displacements of target surfaces along their normal when they are rotating (because of wobble and/or warp of the surface) are measured to be \pm 0.5 to $\pm 2.0 \mu \mathrm{m}$ by the Mitutoyo 513-205 dial indicator. The displacement makes the measured horizontal size larger. But this amount of the displacement is far from sufficient to explain the elliptical shape, particularly for an optical-quality glass target, where surface wobble is $< \pm 0.2 \mu \mathrm{m}$ (our estimated detection limit), but where we still see elliptical source dimensions. In order to 


\begin{tabular}{lccccc}
\hline Element & $\begin{array}{c}\text { Total X-ray } \\
\left(10^{-6}\right)\end{array}$ & $\begin{array}{c}K_{\alpha} \text { X-ray } \\
\left(10^{-6}\right)\end{array}$ & $\begin{array}{c}K_{\alpha}+K_{\beta} \text { X-ray } \\
\left(10^{-6}\right)\end{array}$ & $\begin{array}{c}\text { Electron temperature } \\
(\mathrm{keV})\end{array}$ & $\begin{array}{c}\text { Measured source size } \\
\text { horizontal } \times \text { vertical } \\
\mu \mathrm{m} \times \mu \mathrm{m})\end{array}$ \\
\hline $\mathrm{Cu}$ & 45.21 & 17.10 & 20.78 & 18.55 & $(10.3 \pm 0.8) \times(12.5 \pm 1.0)$ \\
$\mathrm{Ge}$ & 46.30 & 18.01 & 22.74 & 17.01 & $(9.0 \pm 0.7) \times(10.4 \pm 1.4)$ \\
$\mathrm{Mo}$ & 211.9 & 40.83 & 57.07 & 19.91 & $(7.3 \pm 0.4) \times(11.4 \pm 1.7)$ \\
$\mathrm{Ag}$ & 208.6 & 30.68 & 46.62 & 33.99 & $(10.2 \pm 0.7) \times(15.0 \pm 1.2)$ \\
$\mathrm{Sn}$ & 94.20 & 12.79 & 21.65 & 47.89 & $\mathrm{~A}$ \\
\hline
\end{tabular}

TABLE 1 Measured source size, electron temperature, and energy conversion efficiencies from laser to total X-ray emission, and from laser to characteristic lines, calculated from measured spectral data

determine the effect of polarization on the source size, we measured the source sizes for $s$-polarized pump light, and find that the sizes are smaller than $p$-polarized pumped X-ray sources and they are still elliptical. In addition, the laser is incident on the target at a $45^{\circ}$ incident-angle in our experimental geometry. The laser spot on the target surface is elliptical because of projection. But we measure the source size at a $40^{\circ}$ angle on other side of the target normal. This nearly cancels the projection effect. The energy transport mechanism that gives rise to the elliptical X-ray source shape warrants further study.

The measured vertical X-ray source sizes are 6.1-8.6 times larger than the laser focal spot size. In non- $\lambda^{3}$ experiments [40] X-ray source sizes may be 2-4 times larger than their pump laser focal spot. The lateral movement of suprathermal electrons during the X-ray generation is the main reason for this effect. The laser energy is transferred to the electrons in the plasma through the processes of resonance absorption, vacuum heating, and $\boldsymbol{J} \times \boldsymbol{B}$ heating. These electrons can move significant distances in lateral direction during X-ray generation, or can escape from the target surface and reenter the bulk material due to electrostatic force at a significant distance from the laser spot. This produces larger X-ray source size. In particular, for the $\lambda^{3}$ laser regime, the laser intensity gradient is larger, and the ponderomotive force is more three-dimensional and stronger compared with experiments using non- $\lambda^{3}$ laser. Thus, this lateral effect must be more pronounced in our experiments. Consequently, for the wavelength-scale focal spot, the ratio between the X-ray source size and laser spot size is larger.

To quantify the off-peak effects, we measured the transverse intensity profile of our laser at the target using a 16bit CCD camera. Focal spot images are shown in Fig. 3. Image (a) is a regular focal spot after deformable mirror is optimized. We can see that it has a smooth elliptical shape. The FWHM of the spot is $1.2 \times 1.4 \mu \mathrm{m}^{2}$ and the peak intensity is $\sim 2.2 \times 10^{18} \mathrm{~W} / \mathrm{cm}^{2} .35 .0 \%$ of pulse energy is focused in the FWHM area, and $64.7 \%$ is included in the area of $2.0 \times$ $2.4 \mu \mathrm{m}^{2}\left(1 / \mathrm{e}^{2}\right)$. In order to see the shoulder distribution of the focal spot, the contrast and brightness of the image shown in Fig. 3a are changed, and the modified image is shown in Fig. 3b. The most intense three lobes around the main spot, $\mathrm{A}$, are marked as B, C and D. Their intensities are $1.2 \times 10^{17}$, $1.3 \times 10^{17}$, and $5.7 \times 10^{16} \mathrm{~W} / \mathrm{cm}^{2}$, respectively. For B, C, D individual lobes, they contain $2.7 \%, 6.5 \%$ and $1.1 \%$ of the laser pulse energy within the $1 / \mathrm{e}^{2}$ spot. The distances between main spot and the lobes are $2.2 \mu \mathrm{m}(\mathrm{AD}), 2.0 \mu \mathrm{m}(\mathrm{AB})$, and $3.5 \mu \mathrm{m}(\mathrm{AC})$.
A higher X-ray yield in the laser-solid interaction is not necessarily generated from the highest laser intensity. The low-intensity wings of the laser spot on target may generate X-rays more efficiently, according to Eder's model [28] and Reich's model [41], which report similar intensities to those found in our lobes. For lobe intensities in our experiments, they generate X-ray few times more efficiently than the main spot intensity. However, the lobes intensities are 17-39 times lower than the main spot intensity. So, the FWHM X-ray source size is determined by the main laser spot, not the lobes. Furthermore, each lobe contains only few percent of laser pulse energy, 10-59 times lower than the energy in the main spot. Their contribution to the total X-ray yield is small. Therefore, existence of the lobes is not the main reason for the fact that the X-ray source size is much larger than the laser spot size and the X-ray source is elliptical. Also we can see that the lobes are almost located near the vertical axis, but the vertical size of X-ray source is smaller than the horizontal.

By our $\lambda^{3}$ laser, $\sim 10 \mu \mathrm{m} \mathrm{X-ray} \mathrm{sources} \mathrm{are} \mathrm{generated.}$ The smaller the source size, the better the spatial coherence. Our X-ray diffraction experiments with D. Boschetto and $\mathrm{A}$. Rousse using a Si target indicate better spatial coherence in the vertical dimension. This is consistent with an ellipticity on the order of those measured here. The small dimensions of our hard X-ray sources are also critical to the improvement of imaging applications. The high repetition rate inherently in the relativistic $\lambda^{3}$ regime further promotes the utility of these sources. It is expected that they will provide significant enhancement of image quality in medical applications. In particular, microscopic $17-25 \mathrm{keV} \mathrm{Mo}$, $\mathrm{Ag}$, and Sn sources are ideally suited for high-resolution mammography.

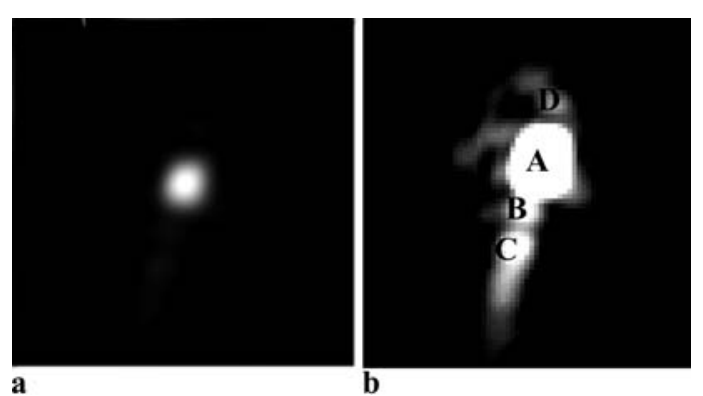

FIGURE 3 (a) A 16-bit CCD image of the focal spot after the deformable mirror is optimized (b) Modified image in order to see the shoulder distribution 
5

\section{Summary}

In conclusion, we report the results on laser-matter interaction in the relativistic $\lambda^{3}$ regime. Hard X-ray spectroscopy of several metallic targets, including $\mathrm{Cu}, \mathrm{Ge}, \mathrm{Mo}$, $\mathrm{Ag}$, and $\mathrm{Sn}$, has been experimentally studied under relativistic intensity using a compact tabletop laser at high repetition rate. The $K_{\alpha}$ and $K_{\beta}$ peaks of all targets were observed. Electron temperatures and conversion efficiencies (from laser to $\mathrm{X}$-rays) are calculated from the experimental data. The X-ray source size is measured to be $\sim 10$ micron, and the source shape is elliptical. Further investigation of source shape and its dependence of focal intensity distribution is found to be warranted.

ACKNOWLEDGEMENTS The authors wish to thank Dr. A. Rousse and Dr. W. Theobald for helpful discussions. This work was supported by the National Science Foundation through the Frontiers of Physics Program at the Center for Ultrafast Optical Science (FOCUS \#PHY0114336) and the Michigan Economic Development Corporation's MLSC (\#0085P1001461)

\section{REFERENCES}

1 M.D. Perry, G. Mourou, Science 64, 917 (1994)

2 G.A. Mourou, C.P.J. Barty, M.D. Perry, Phys. Today 51, 22 (1998)

3 S.P.D. Mangles, C.D. Murphy, Z. Najmudin, A.G.R. Thomas, J.L. Collier, A.E. Dangor, E.J. Divall, P.S. Foster, J.G. Gallacher, C.J. Hooker, D.A. Jaroszynski, A.J. Langley, W.B. Mori, P.A. Norreys, F.S. Tsung, R. Viskup, B.R. Walton, K. Krushelnick, Nature 431, 535 (2004)

4 C.G.R. Geddes, C. Toth, J. van Tilborg, E. Esarey, C.B. Schroeder, D. Bruhwiler, C. Nieter, J. Cary, W.P. Leemans, Nature 431, 538 (2004)

5 J. Faure, Y. Glinec, A. Pukhov, S. Kiselev, S. Gordienko, E. Lefebvre, J.-P. Rousseau, F. Burgy, V. Malka, Nature 431, 541 (2004)

6 D. Umstadter, S.-Y. Chen, A. Maksimchuk, G. Mourou, R. Wagner, Science 273, 472 (1996)

7 A.G. Zhidkov, A. Sasaki, T. Tajima, T. Auguste, P. D’Olivera, S. Hulin, P. Monot, A.Y. Faenov, T.A. Pikuz, I.Y. Skobelev, Phys. Rev. E 60, 3273 (1999)

8 A. Maksimchuk, S. Gu, K. Flippo, D. Umstadter, V.Y. Bychenkov, Phys. Rev. Lett. 84, 4108 (2000)

9 J.D. Kmetec, C.L. Gordon III, J.J. Macklin, B.E. Lemoff, G.S. Brown, S.E. Harris, Phys. Rev. Lett. 68, 1527, (1992)

10 J.C. Kieffer, J.P. Matte, H. Pépin, M. Chaker, Y. Beaudoin, T.W. Johnston, C.Y. Chien, S. Coe, G. Mourou, J. Dubau, Phys. Rev. Lett. 68, 480 (1992)

11 A. Rousse, K.T. Phuoc, R. Shah, A. Pukhov, E. Lefebvre, V. Malka, S. Kiselev, F. Burgy, J.-P. Rousseau, D. Umstadter, D. Hulin, Phys. Rev. Lett. 93, 135005 (2004)

12 F.N. Beg, A.R. Bell, A.E. Dangor, C.N. Danson, A.P. Fews, M.E. Glinsky, B.A. Hammel, P. Lee, P.A. Norreys, M. Tatarakis, Phys. Plasmas 4, 447 (1997)

13 P.A. Norreys, M. Santala, E. Clark, M. Zepf, I. Watts, F.N. Beg, K. Krushelnick, M. Tatarakis, A.E. Dangor, X. Fang, P. Graham, T. McCanny, R.P. Singhal, K.W.D. Ledingham, A. Creswell, D.C.W. Sander- son, J. Magill, A. Machacek, J.S. Wark, R. Allott, B. Kennedy, D. Neely, Phys. Plasmas 6, 2150 (1999)

14 J.W. Shearer, J. Garrison, J. Wong, J.E. Swain, Phys. Rev. A 8, 1582 (1973)

15 T.E. Cowan, A.W. Hunt, T.W. Phillips, S.C. Wilks, M.D. Perry, C. Brown, W. Fountain, S. Hatchett, J. Johnson, M.H. Key, T. Parnell, D.M. Pennington, R.A. Snavely, Y. Takahashi, Phys. Rev. Lett. 84, 903 (2000)

16 C.E. Max, J. Arons, A.B. Langdon, Phys. Rev. Lett. 33, 209 (1974)

17 S.-Y. Chen, G.S. Sarkisov, A. Maksimchuk, R. Wagner, D. Umstadter, Phys. Rev. Lett. 80, 2610 (1998)

18 R. Lichters, J. Meyer-ter-Vehn, A. Pukhov, Phys. Plasmas 3, 3425 (1996)

19 S.C. Wilks, W.L. Kruer, IEEE J. Quantum Electron. QE-33, 1954 (1997)

20 J.C. Kieffer, M. Chaker, J.P. Matte, H. Pepin, C.Y. Côté, Y. Beaudoin, T.W. Johnston, C.Y. Chien, S. Coe, G. Mourou, O. Peyrusse, Phys. Fluids B 5, 2676 (1993)

21 C. Rischel, A. Rousse, I. Uschmann, P.-A. Albouy, J.-P. Geinder, P. Audebert, J.-C. Gauthier, E. Froster, J.-L. Martin, A. Antonetti, Nature 390, 490 (1997)

22 A. Rousse, A. Antonetti, P. Audebert, A. Dos Santos, F. Fallies, J.P. Geindre, G. Grillon, A. Mysyrowicz, J.C. Gauthier, J. Phys. B 27, L697 (1994)

23 F. Raksi, K.R. Wilson, Z. Jiang, A. Ikhlef, C.Y. Côté, J.-C. Kieffer, J. Chem. Phys. 104, 6066 (1996)

24 A. Krol, A. Ikhlef, J.C. Kieffer, D.A. Bassano, C.C. Chamberlain, Z. Jiang, H. Pe'pin, S.C. Prasad, Med. Phys. 24, 725, (1997)

25 A. Rousse, C. Rischel, S. Fourmaux, I. Uschmann, S. Sebban, G. Grillon, P. Balcou, E. Förster, J.P. Geindre, P. Audebert, J.C. Gauthier, D. Hulin, Nature 410, 65, (2001)

26 N.M. Naumova, J.A. Nees, I.V. Sokolov, B. Hou, G.A. Mourou, Phys. Rev. Lett. 92, 063902 (2004)

27 N. Naumova, I. Sokolov, J. Nees, A. Maksimchuk, V. Yanovsky, G. Mourou, Phys. Rev. Lett. 93, 195003 (2004)

28 D.C. Eder, G. Pretzler, E. Fill, K. Eidmann, A. Saemann, Appl. Phys. B 70, $211(2000)$

29 G. Pretzler, T. Schlegel, E. Fill, D. Eder, Phys. Rev. E 62, 5618 (2000)

30 T. Guo, C. Spielmann, B.C. Walker, C.P.J. Barty, Rev. Sci. Instrum. 72, $41(2001)$

31 F. Ewald, H. Schwoerer, R. Sauerbrey, Europhys. Lett. 60, 710 (2002)

32 A. Sjögren, M. Harbst, C.-G. Wahlstrom, S. Svanberg, C. Olsson, Rev. Sci. Instrum. 74, 2300 (2003)

33 Y. Jiang, T. Lee, W. Li, G. Ketwaroo, C.G. Rose-Petruck, Opt. Lett. 27, 963 (2002)

34 G. Korn, A. Thoss, H. Stiel, U. Vogt, M. Richardson, T. Elsaesser, M. Faubel, Opt. Lett. 27, 866 (2002)

35 O. Albert, H. Wang, D. Liu, Z. Chang, G. Mourou, Opt. Lett. 25, 1125 (2000)

36 D. Liu, J. Nees, H. Wang, G. Mourou, Z. Chang, Proceedings of the Conference on Lasers and Electrooptics, San Francisco, PD5/667-668 (2000)

37 I.H. Hutchinson, Principles of Plasma Diagnostics (Cambridge University Press, 1987)

38 A. Thompson, X-ray Data Booklet. Lawerence Berkeley National Laboratory, Berkeley CA (2001) (presently available at http://xdb.lbl.gov)

39 G. Kulcsár, D. AlMawlawi, F.W. Budnik, P.R. Herman, M. Moskovits, L. Zhao, R.S. Marjoribanks, Phys. Rev. Lett. 84, 5149 (2000)

40 J. Yu, Z. Jiang, J.C. Kieffer, A. Krol, Phys. Plasmas 6, 1318 (1999)

41 C. Reich, P. Gibbon, I. Uschmann, E. Förster, Phys. Rev. Lett. 84, 4846 (2000) 